\title{
Performance of solid and liquid culture media for the detection of Mycobacterium tuberculosis in clinical materials: meta-analysis of recent studies
}

\author{
F. Rageade $\cdot$ N. Picot $\cdot$ A. Blanc-Michaud $\cdot$ S. Chatellier . \\ C. Mirande • E. Fortin • A. van Belkum
}

Received: 25 March 2014 / Accepted: 31 March 2014 / Published online: 24 April 2014

(C) Springer-Verlag Berlin Heidelberg 2014

Tuberculosis (TB) is a universal disease: it occurs all over the globe, albeit with hugely varying regional incidence. Especially in Asian and African countries, the incidence and prevalence may be extremely high. The European Journal of Clinical Microbiology and Infectious Diseases published seven papers on TB in 2013, representing $3.3 \%$ (7/211) of its annual production. Four of the papers concerned mechanistic studies on clinical patient management, cost-effectiveness of antibiotic treatment, importance of microbiota and host genetic polymorphisms, respectively. The other three papers concerned molecular diagnostics, so, overall, hardly any attention was paid to the more classical modes of diagnosing TB. And this clearly contrasts with international needs, since classical diagnostics is still very high on the agenda in those countries where TB testing is most needed. In their 2013 Global Tuberculosis Report, the World Health Organization (WHO) claimed that culture is still the reference method for the detection of Mycobacterium tuberculosis (Mtb), with smear staining and microscopy being considered as a method of "added value". Hence, a wide array of culture systems has been developed by a large multitude of researchers and companies. Some of these are semi-automated, but most require manual intervention. Löwenstein-Jensen medium (LJ) is frequently considered the key mycobacterial growth medium when comparative studies for the verification and validation of new diagnostic tests are performed. Even the validation of liquid media in combination with semi-automated culture

F. Rageade $\cdot$ N. Picot $\cdot$ A. Blanc-Michaud $\cdot$ S. Chatellier $\cdot$

C. Mirande $\cdot$ E. Fortin $\cdot$ A. van Belkum $(\triangle)$

bioMérieux SA, Chemin de l'Orme, 69280 Marcy l'Etoile, France

e-mail: alex.vanbelkum@biomerieux.com

F. Rageade

e-mail: francoise.rageade@biomerieux.com systems is usually performed in comparison with LJ-based cultivation as the diagnostic Gold Standard.

We have set out to investigate the quality of some of the culture media proposed for the detection of Mtb in order to verify whether such media really are the appropriate Gold Standard tools for the validation of novel TB diagnostics. We performed a literature search (PubMed dd 13 August 2013) for the period between January 2008 and August 2013. In this way, 71 relevant articles were identified, of which the 19 most complete studies were selected for more detailed analysis. We collected the number of specimens tested, the culture medium used and identified, where possible, the manufacturer of the media. As such, six different producers of culture media for Mtb detection were identified. When microscopic observation of drug susceptibility (MODS) was performed, we included these data as well.

Overall, the highest sensitivities and negative predictive values were shown by the BACTEC MGIT 960 System (Becton-Dickinson, Sparks, MD, USA), either when used alone or in combination with LJ. The latter combination provided the best sensitivity and also scored particularly well in the detection of non-tuberculous mycobacteria. The highest specificity and positive predictive values were shown by the LJ method itself, which obviously produced no falsepositives. So, MGIT is the top performer with regard to sensitivity, while LJ sets the current standard for specificity. This is well in line with international findings and recommendations.

As can be seen in Table 1, liquid media show times to positivity that are about half of those for solid media (about 14.2 days for MGIT versus 28 days for $\mathrm{LJ}$ ). However, and this is important, afar-based media from various suppliers show differences in performance, with time to positivity averages ranging from 22.5 to 32 days. Assuming that there were no systematic differences in the bacterial loads of the samples 


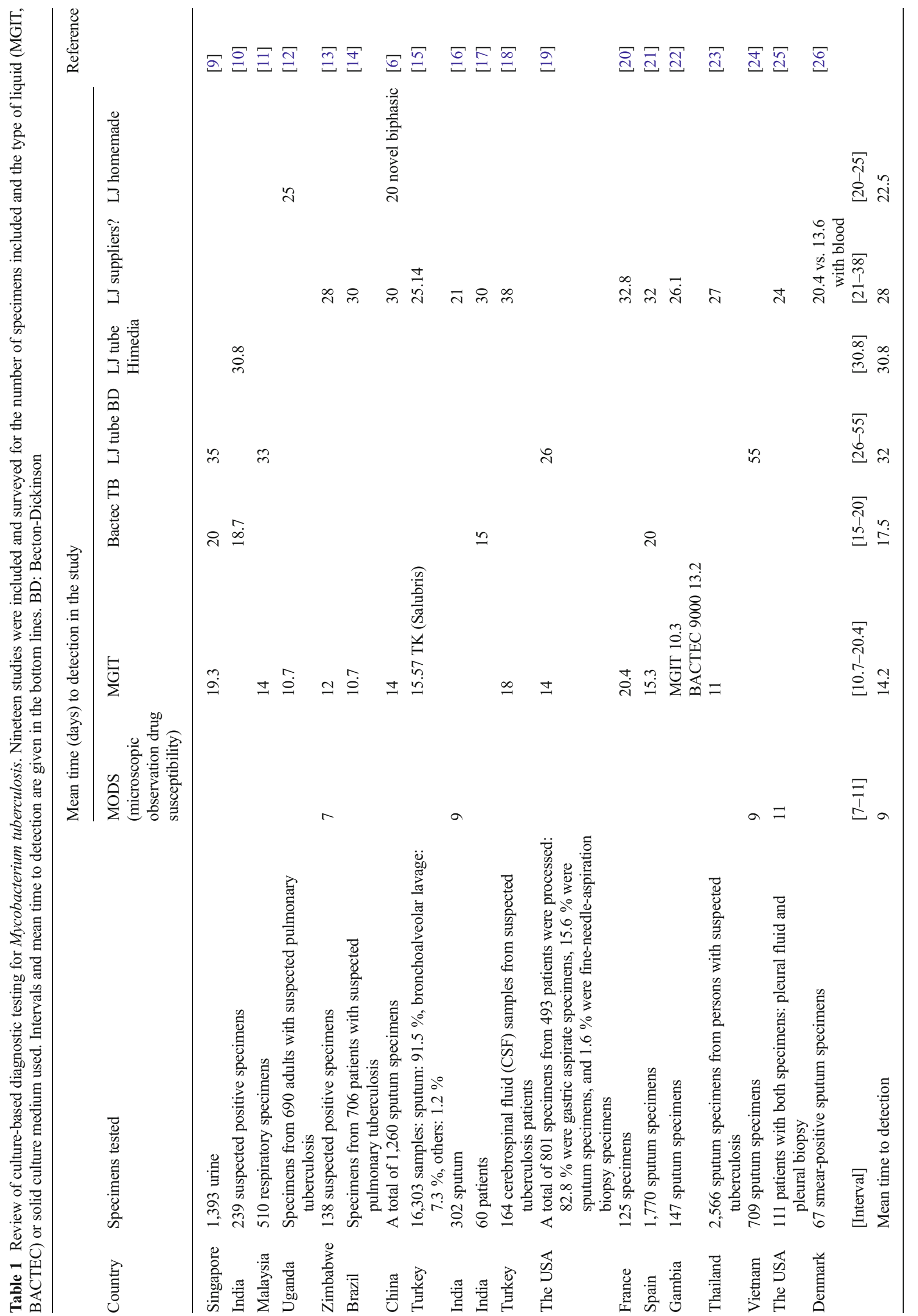


collected during the different studies or in the pre-analytical way in which the samples were processed $[1,2]$, these are differences in timing that could bias validation studies of new diagnostics, depending on where and how and with which LJ media these studies are performed.

In addition, recent developments were noted during the literature review. For instance, a colourimetric nitrate reductase assay (NRA) performed quite well and with short turnaround times [3, 4]. Also, changes of media when resistance testing is involved is worth considering: on Middlebrook $7 \mathrm{H} 11$, the detection of isoniazid and rifampicin resistance is more reliable than on $\mathrm{LJ}[5]$. A recent Chinese study evaluated a novel biphasic culture medium for the recovery of mycobacteria from sputum specimens from suspected pulmonary tuberculosis patients [6]. The system consisted of a 7-ml slant of LJ, $3 \mathrm{ml}$ liquid medium, a chromogenic growth indicator and antimicrobial agents that facilitated rapid screening for drug-resistant Mtb. Times to detection were 14 days, 20 days and 30 days for cultures grown in MGIT, in biphasic medium or on LJ slants, respectively. Tests showed times to positivity of 9.6 and 21.4 days for the biphasic medium versus classical LJ, respectively. Tests performed in Pakistan showed times to positivity of 9.6 and 21.4 days for the biphasic medium versus classical LJ, respectively [6]. The biphasic medium does not require expensive detection instrumentation and it was expected to be a useful alternative for other forms of mycobacterial culture, especially in hospitals that lack TBcompatible culture equipment. The addition of blood in the media is important and a Danish study from 2009 showed that rapid culture of Mtb on blood agar in resource-limited settings reduced the mean time to detection of mycobacteria from 20.4 to 13.6 days in an LJ-type medium.

In conclusion, culture remains the key technology in the diagnosis of TB, despite emerging new technologies, including polymerase chain reaction (PCR) or other methods [7, 8]. However, also, culture needs to be subjected to thorough quality assessment locally, given the large differences in time to positivity that can be distilled from the international literature, even when a well-accepted culture medium such as LJ is being considered. Compliance with the quality assessment may already lead to clinically valuable improvements in the quality of diagnosis and, hence, the quality of care for TB patients.

\section{References}

1. Zingué D, Hien H, Méda N, Zida S, Kaboré A, Sanou A, Ouédraogo AS, Gomgnimbou M, Diandé S, Tarnagda Z, Godreuil S (2013) Advantages and drawbacks of expectoration decontamination methods for tuberculosis and anti-tuberculosis drug resistance diagnosis. Ann Biol Clin (Paris) 71:283-291
2. Chatterjee M, Bhattacharya S, Karak K, Dastidar SG (2013) Effects of different methods of decontamination for successful cultivation of Mycobacterium tuberculosis. Indian J Med Res 138:541-548

3. Coban AY, Deveci A, Sunter AT, Martin A (2014) Nitrate reductase assay for rapid detection of isoniazid, rifampin, ethambutol, and streptomycin resistance in Mycobacterium tuberculosis: a systematic review and meta-analysis. J Clin Microbiol 52:15-19

4. Rojas-Ponce G, Rachow A, Guerra H, Mapamba D, Joseph J, Mlundi R, Marimoto S, Ntinginya NE, Mangu C, Framhein A, Butler A, Kohlenberg A, Ngatemelela D, Froeschl G, Maboko L, Hoelscher M, Heinrich N (2013) A continuously monitored colorimetric method for detection of Mycobacterium tuberculosis complex in sputum. Int J Tuberc Lung Dis 17:1607-1612

5. Satti L, Ikram A, Palomino JC, Martin A, Khan FA (2013) Field evaluation of the direct detection of multidrug resistant Mycobacterium tuberculosis by nitrate reductase assay on 7H11 agar. Tuberculosis (Edinb) 93:308-311

6. Cui Z, Wang J, Zhu C, Huang X, Lu J, Wang Q, Chen Z, Wang J, Zhang Y, Gu D, Jing L, Chen J, Zheng R, Qin L, Yang H, Jin R, Liu Z, Bi A, Liu J, Hu Z (2012) Evaluation of a novel biphasic culture medium for recovery of mycobacteria: a multi-center study. PLoS One 7:e36331

7. Leslie D (2014) Molecular methods for mycobacteria. Pathology 46: S46

8. Costa MN, Veigas B, Jacob JM, Santos DS, Gomes J, Baptista PV, Martins R, Inácio J, Fortunato E (2014) A low cost, safe, disposable, rapid and self-sustainable paper-based platform for diagnostic testing: lab-on-paper. Nanotechnology 25:094006

9. Chan DS, Choy MY, Wang S, Sng LH (2008) An evaluation of the recovery of mycobacteria from urine specimens using the automated Mycobacteria Growth Indicator Tube system (BACTEC MGIT 960). J Med Microbiol 57:1220-1222

10. Naveen G, Peerapur BV (2012) Comparison of the LowensteinJensen Medium, the Middlebrook 7H10 Medium and MB/BacT for the Isolation of Mycobacterium tuberculosis (MTB) from clinical specimens. J Clin Diagn Res 6:1704-1709

11. Fadzilah MN, Ng KP, Ngeow YF (2009) The manual MGIT system for the detection of M. tuberculosis in respiratory specimens: an experience in the University Malaya Medical Centre. Malays J Pathol 31:93-97

12. Boum Y 2nd, Orikiriza P, Rojas-Ponce G, Riera-Montes M, Atwine D, Nansumba M, Bazira J, Tuyakira E, De Beaudrap P, Bonnet M, Page AL (2013) Use of colorimetric culture methods for detection of Mycobacterium tuberculosis complex isolates from sputum samples in resource-limited settings. J Clin Microbiol 51:2273-2279

13. Makamure B, Mhaka J, Makumbirofa S, Mutetwa R, Mupfumi L, Mason P, Metcalfe JZ (2013) Microscopic-observation drugsusceptibility assay for the diagnosis of drug-resistant tuberculosis in Harare, Zimbabwe. PLoS One 8:e55872

14. Moreira AS, Huf G, Vieira MA, Fonseca L, Ricks M, Kritski A (2013) Performance comparison between the mycobacteria growth indicator tube system and Löwenstein-Jensen medium in the routine detection of Mycobacterium tuberculosis at public health care facilities in Rio de Janeiro, Brazil: preliminary results of a pragmatic clinical trial. J Bras Pneumol 39:365-367

15. Kocagöz T, Altın S, Türkyılmaz Ö, Taș İ, Karaduman P, Bolaban D, Yeșilyurt E, Öktem S, Aytekin N, Șınık G, Mozioğlu E, Silier T (2012) Efficiency of the TK Culture System in the diagnosis of tuberculosis. Diagn Microbiol Infect Dis 72:350-357

16. Lazarus RP, Kalaiselvan S, John KR, Michael JS (2012) Evaluation of the microscopic observational drug susceptibility assay for rapid and efficient diagnosis of multi-drug resistant tuberculosis. Indian $\mathrm{J}$ Med Microbiol 30:64-68

17. Panicker JN, Nagaraja D, Subbakrishna DK, Venkataswamy MM, Chandramuki A (2010) Role of the BACTEC radiometric method in 
the evaluation of patients with clinically probable tuberculous meningitis. Ann Indian Acad Neurol 13:128-131

18. Thakur R, Goyal R, Sarma S (2010) Laboratory diagnosis of tuberculous meningitis - is there a scope for further improvement? J Lab Physicians 2:21-24

19. Brittle W, Marais BJ, Hesseling AC, Schaaf HS, Kidd M, Wasserman E, Botha T (2009) Improvement in mycobacterial yield and reduced time to detection in pediatric samples by use of a nutrient broth growth supplement. J Clin Microbiol 47:1287-1289

20. Gérôme P, Fabre M, Soler CP, De Pina JJ, Simon F (2009) Comparison of the mycobacteria growth indicator tube with solid culture for the detection of tuberculosis complex mycobacteria from blood. Pathol Biol (Paris) 57:44-50

21. Sorlozano A, Soria I, Roman J, Huertas P, Soto MJ, Piedrola G, Gutierrez J (2009) Comparative evaluation of three culture methods for the isolation of mycobacteria from clinical samples. J Microbiol Biotechnol 19:1259-1264

22. Otu J, Antonio M, Cheung YB, Donkor S, De Jong BC, Corrah T, Adegbola RA (2008) Comparative evaluation of BACTEC MGIT 960 with BACTEC $9000 \mathrm{MB}$ and LJ for isolation of mycobacteria in The Gambia. J Infect Dev Ctries 2:200-205
23. Srisuwanvilai LO, Monkongdee P, Podewils LJ, Ngamlert K, Pobkeeree V, Puripokai P, Kanjanamongkolsiri P, Subhachaturas W, Akarasewi P, Wells CD, Tappero JW, Varma JK (2008) Performance of the BACTEC MGIT 960 compared with solid media for detection of Mycobacterium in Bangkok, Thailand. Diagn Microbiol Infect Dis 61:402-407

24. Dang TM, Nguyen TN, Wolbers M, Vo SK, Hoang TT, Nguyen HD, To MH, Vuong MB, Nguyen TP, Tran VQ, Nguyen TB, Vo TH, Nguyen TN, Dai VH, Phan TH, Nguyen HD, Farrar J, Caws M (2012) Evaluation of microscopic observation drug susceptibility assay for diagnosis of multidrug-resistant tuberculosis in Viet Nam. BMC Infect Dis $12: 49$

25. Tovar M, Siedner MJ, Gilman RH, Santillan C, Caviedes L, Valencia T, Jave O, Escombe AR, Moore DA, Evans CA (2008) Improved diagnosis of pleural tuberculosis using the microscopic-observation drug-susceptibility technique. Clin Infect Dis 46:909-912

26. Mathur ML, Gaur J, Sharma R, Solanki A (2009) Rapid culture of Mycobacterium tuberculosis on blood agar in resource limited setting. Dan Med Bull 56:208-210 